\title{
Characterization by Confocal Laser Scanning Microscopy of the Phase Composition at Interfaces in Thick Films of Polymer Blends
}

\author{
Sandro Lattante, ${ }^{1}$ Andrea Perulli, ${ }^{2}$ and Marco Anni ${ }^{1}$ \\ ${ }^{1}$ Dipartimento di Matematica e Fisica "Ennio De Giorgi", Universitá del Salento, via per Arnesano, 73100 Lecce, Italy \\ ${ }^{2}$ Dipartimento di Ingegneria dell'Innovazione, Universitá del Salento, via per Monteroni, 73100 Lecce, Italy \\ Correspondence should be addressed to Sandro Lattante; sandro.lattante@unisalento.it
}

Received 27 December 2013; Revised 25 February 2014; Accepted 26 February 2014; Published 30 March 2014

Academic Editor: Cornelia Vasile

Copyright (c) 2014 Sandro Lattante et al. This is an open access article distributed under the Creative Commons Attribution License, which permits unrestricted use, distribution, and reproduction in any medium, provided the original work is properly cited.

Confocal Laser Scanning Microscopy (CLSM) has been used as a fast, user-friendly, and noninvasive tool for characterizing the phase composition differences at the substrate and air interfaces in thick films of polymer blends. A clearly different phase composition at the blend/glass interface and at the blend/air interface has been detected. We show that PCBM preferentially accumulates at the glass/blend interface, while P3HT preferentially accumulates at the blend/air interface, by comparing the integrated signal intensity of the luminescence coming from both interfaces. Our results demonstrate that CLSM can be used conveniently for the fast identification of a preferential phase segregation at interfaces in polymer blends. This is useful in the research field on devices (like sensors or planar waveguides) that are based on very thick layers (thickness higher than $1 \mu \mathrm{m}$ ).

\section{Introduction}

In the last few years, it has been widely demonstrated, using several complex experimental techniques, that many systems composed of organic blends undergo a vertical phase stratification. For instance, polymers blended with fullerenes like poly[(9,9-dioctylfluorenyl-2,7-diyl)-alt-5,5(40,70-di-2-thienyl-20,10,30-benzothiadiazole)] (APFO-3) $[1,2]$ where the dynamic secondary ion mass spectroscopy (SIMS) technique has been used for the characterization, or poly $\left[\left(4,4^{\prime}\right.\right.$-bis(2-ethylhexyl)dithieno[3,2-b:2 $2^{\prime}, 3^{\prime}$-d] silole)2,6-diyl-alt-(2,1,3-benzothiadiazole)-4,7-diyl] (PSBTBT) [3] using XRD and small-angle Neutron Scattering, or Poly[[9(1-octylnonyl)-9H-carbazole-2,7-diyl]-2,5-thiophenediyl-2,1, 3-benzothiadiazole-4,7-diyl-2,5-thiophenediyl] (PCDTBT) [4] as well as all-polymer blends like poly(9,9-di-noctylfluorene-alt-benzothiadiazole) (F8BT) and poly(9,9di-n-octylfluorene-alt-(1,4-phenylene-((4-sec-butylphenyl) imino)-1,4-phenylene)) (TFB) [5]. The vertical stratification of the prototype poly(3-hexylthiophene) (P3HT) blended with $[6,6]$-phenyl-C61-butyric acid methyl ester (PCBM) has been characterized by several experimental techniques. Tsoi et al. studied thin films of RR-P3HT: PCBM and RRaP3HT : PCBM by UV and X-ray photoelectron spectroscopy (UPS and XPS) [6], demonstrating that the P3HT chains are preferentially ordered at the blend-air interface, while PCBM tends to accumulate at the substrate-blend interface. Xue et al. [7] investigated the effect of the annealing process on the vertical stratification of P3HT: PCBM blend by means of X-ray diffraction (XRD) and near-edge X-ray absorption fine structure spectroscopy (NEXAFS). Karagiannidis et al. [8] also studied the annealing effects on the vertical phase rearrangement in P3HT: $\mathrm{PCBM}$ thin films by XRD, confirming the accumulation of PCBM on the bottom interface and of $\mathrm{P} 3 \mathrm{HT}$ at the top interface. Ellipsometry has been used too by Madsen and coworkers [9] for monitoring the vertical phase separation in P3HT: PCBM blend, finding results compatible with that given by XPS characterization.

Confocal Laser Scanning Microscopy (CLSM) can not attain the spatial resolution proper of the above mentioned techniques (in the nm scale), being limited by the diffraction of light. Moreover, it can not be used for a morphological 
vertical gradient characterization of very thin films (like the ones usually employed in fields such as Organic Light Emitting Diodes or Polymer Solar Cells [10]). Nevertheless, it is a simple, time-preserving, diffused and user friendly technique to be exploited for a fast and preliminary check of experimental parameters that can be of importance in designing a particular experiment where a very thick $(\geq 1 \mu \mathrm{m})$ polymer film is desirable. For instance, the preferential phase segregation at interfaces in polymer blends is a key parameter to be known in order to properly understand and characterize the physics and behavior of organic sensor or planar waveguides where a thick organic layer is employed. For example the ideal thickness of bulk heterojunction organic based photodiodes for image sensing should be $\sim 4 \mu \mathrm{m}$ in order to drastically reduce the reverse bias dark current [11]. Thick films $(\sim 1 \mu \mathrm{m})$ are also needed to obtain high illuminance detection from ink-jet printed photosensors based on thick luminescent polymer blends [12]. Since the usual device structure is the diode-like one (where the active layer is sandwiched between two conductor layers), it should be desirable to know if there is a difference in the blend composition at the interfaces in order to choose the proper top and bottom conductive layers, improving the charge injection/extraction by matching the interfaces between the hole (electron) injection/extraction conductive layer with the hole (electron) transporting phase inside the active layer blend. Thick samples are also requested when studying the waveguide-assisted Amplified Spontaneous Emission (ASE) from organic layers composed of luminescent species embedded into polymer inert matrix [13], or lasing effects on thick bilayers [14]. The optical gain in the waveguide is strongly dependent on the thickness of the active layer and on the overlap between the gain media, the pump laser beam, and the electromagnetic field inside the waveguide [15-17]. Therefore, it should be desirable to know if there is an accumulation on one side of the sample of the luminescent phase inside the wave-guiding layer for thicknesses in the micrometric range.

Although P3HT: $\mathrm{PCBM}$ is a standard system used in the field of organic solar cells, here it has been chosen as a mere testing system for the proposed technique, due to the fact that it is already well known that a thick blend of P3HT: $\mathrm{PCBM}$ undergoes a vertical phase segregation during the film deposition process, typically forming a thin fullerene-rich layer at the bottom interface and a polymerrich layer at the top interface [18]. We point out that we have no intention to extend our findings to solar energy conversion based on organic thin films. Moreover, most of the cited results, related on very thin films, can not be extended by extrapolation to thicker films, for it is known that the phase morphology inside the layer strongly depends on the increasing thickness, up to the micrometric range [19].

The CLSM allows to detect and discriminate the photoluminescence (PL) coming from the substrate/blend and from the blend/air interfaces. Comparing the average PL intensity for each phase at the two interfaces, one can easily and quickly detect if there are component differences at substrate and air interfaces and at what extent, that is the aim of this work.

\section{Materials and Methods}

2.1. Samples Preparation. Three chlorobenzene solutions of P3HT:PCBM have been realized with different relative component ratio, namely $1: 2,1: 1$, and $2: 1$ (in $\mathrm{P} 3 \mathrm{HT}$ relative content increasing order), each solution with a total concentration of $25 \mathrm{mg} / \mathrm{mL}$. Highly regioregular P3HT (Sepiolid P200) has been purchased from Rieke Metals and 99.5\% purity PCBM has been purchased from SES Research, materials have been used as received. The solutions have been stirred for several days at room condition in dark before using them for the thick films preparation.

Ultrathin microscopy cover glass slides (from Bresser, $22 \times 22 \mathrm{~mm}$, certified thickness of $130-170 \mu \mathrm{m}$ ) have been carefully cleaned in an ultrasonic bath sequentially with acetone (10 $\mathrm{min})$, ethanol (10 $\mathrm{min})$, isopropanol $(10 \mathrm{~min})$, and distilled water $(10 \mathrm{~min})$ and then gently dried by a nitrogen gun. Part of the cleaned slides has been monitored by optical microscopy $(40 \mathrm{x})$ in order to check for possible damages due to the cleaning process, no damages have been detected. Few drops of the solutions have been gently placed onto the glass slides inside a Petri dish. The samples were then partly covered in order to protect them from external perturbations. The solutions have been used as stirred for the unfiltered samples realization, while they have been filtered by a PTFE filter $(0.45 \mu \mathrm{m}$ pore size $)$ for the filtered samples. The filtering process allows obtaining samples without macroscopic features that can be easily identified in a photoluminescence map, thus giving the opportunity to test the technique also for very uniform systems. The samples have been left in place few hours until all the solvent was evaporated. All the above operations have been performed at room condition inside a chemical fume hood.

2.2. Photoluminescence. Photoluminescence (PL) spectra have been measured by exciting the samples with a $\mathrm{He}$ Cd laser (325 nm wavelength) filtered by a variable neutral density filter. The PL signal was dispersed by a TRIAX 320 monochromator with a $150 \mathrm{~L} / \mathrm{mm}$ diffraction grating blazed at $500 \mathrm{~nm}$ and detected by a Peltier cooled Si-charge coupled device (CCD). The spectral resolution was $\sim 2 \mathrm{~nm}$.

All the above measurements have been performed at room condition.

2.3. Morphological Characterization. Immediately, after the PL measurements, the samples have been morphologically characterized by Confocal Laser Scanning Microscopy with a Nikon Eclipse C1 inverted confocal microscope. A 40x DIC Plan Apochromat objective (0.95 numerical aperture)equipped with a manually adjustable correction ring-has been used. The samples have been excited by the $488 \mathrm{~nm}$ line of an argon laser and the PL signal has been detected by a couple of photomultipliers (PMTs). The signal was detected simultaneously at $530 \pm 20 \mathrm{~nm}$ using a bandpass filter and above $770 \mathrm{~nm}$ using a high pass filter. Incident excitation power was set to $28 \mu \mathrm{W}$ measured by a power meter placed on the objective focal plane. Single pixel acquisition time was fixed to $42 \mu \mathrm{s}$. 
TABLE 1: Samples average thickness (range bar: $\pm 0.3 \mu \mathrm{m}$ ). P3HT : PCBM ratio in increasing P3HT relative content.

\begin{tabular}{lcc}
\hline Sample $1: 2$ & Sample 1:1 & Sample 2:1 \\
\hline & Unfiltered samples \\
$1.1 \mu \mathrm{m}$ & $1.1 \mu \mathrm{m}$ & $1.2 \mu \mathrm{m}$ \\
\hline \multicolumn{3}{c}{ Filtered samples } \\
$1.2 \mu \mathrm{m}$ & $4 \mu \mathrm{m}$ & $1.2 \mu \mathrm{m}$ \\
\hline
\end{tabular}

Samples thicknesses have been measured by a Dektak Alpha Step profilometer after all the optical and microscopy experiments, thus preventing any preliminary sample damage and/or manipulation. Multiple scratches have been realized all over the samples and thickness values obtained for each scratch have been then averaged. The mean thickness values are reported in Table 1.

In order to characterize the air/blend interface and the glass/blend interface, the samples have been simply tipped over in between the measurement, carefully placing the sample in such a way that the same region is analyzed on both the surfaces. Anyway, no detectable differences have been revealed repeating the measurements on different regions of the given surface, being the sample morphology and thickness homogeneous. Thus, the "tipping over" is not an intrinsic limitation since image shifts are not correlated with any measurable differences in the results. Objective focus has been checked and adjusted at every measurement. During each CLSM experiment, the air/blend interface and the glass/blend interface have been measured keeping constant all the measurement parameters (PMT gain, incident laser power, magnification, and pixel dwell time).

\section{Results and Discussion}

\subsection{Photoluminescence}

Unfiltered Samples. PL spectra of the unfiltered samples are reported in Figure 1, normalized to the film thicknesses. The PL peaks at around $650 \mathrm{~nm}$ and $700 \mathrm{~nm}$ are typical of the P3HT emission [20], while the band around $550 \mathrm{~nm}$ can be attributed to residual PCBM emission due to the intermolecular interactions (absorption and luminescence from charge transfer excited states) between not well separated fullerene clusters [21]. Moreover, there is no evidence of P3HT emission around $550 \mathrm{~nm}$ (pure P3HT PL is also reported as a dashed line in Figure 1, scaled down in order to be comparable with the blend signal). We notice that the relative intensity of the peak at $550 \mathrm{~nm}$ increases with the PCBM content increase, confirming the previous conclusion.

It is well known that the strong PL quenching in this system is related to the efficient charge transfer from $\mathrm{P} 3 \mathrm{HT}$ to PCBM after excitation [22, 23]. The spectral shape of the samples is almost the same irrespective of the relative concentration ratio. As it should be expected, the major P3HT emission quenching effect has been detected in the $1: 2$ sample (almost all the P3HT phase participate to the charge transfer process to the overwhelming PCBM phase), while on
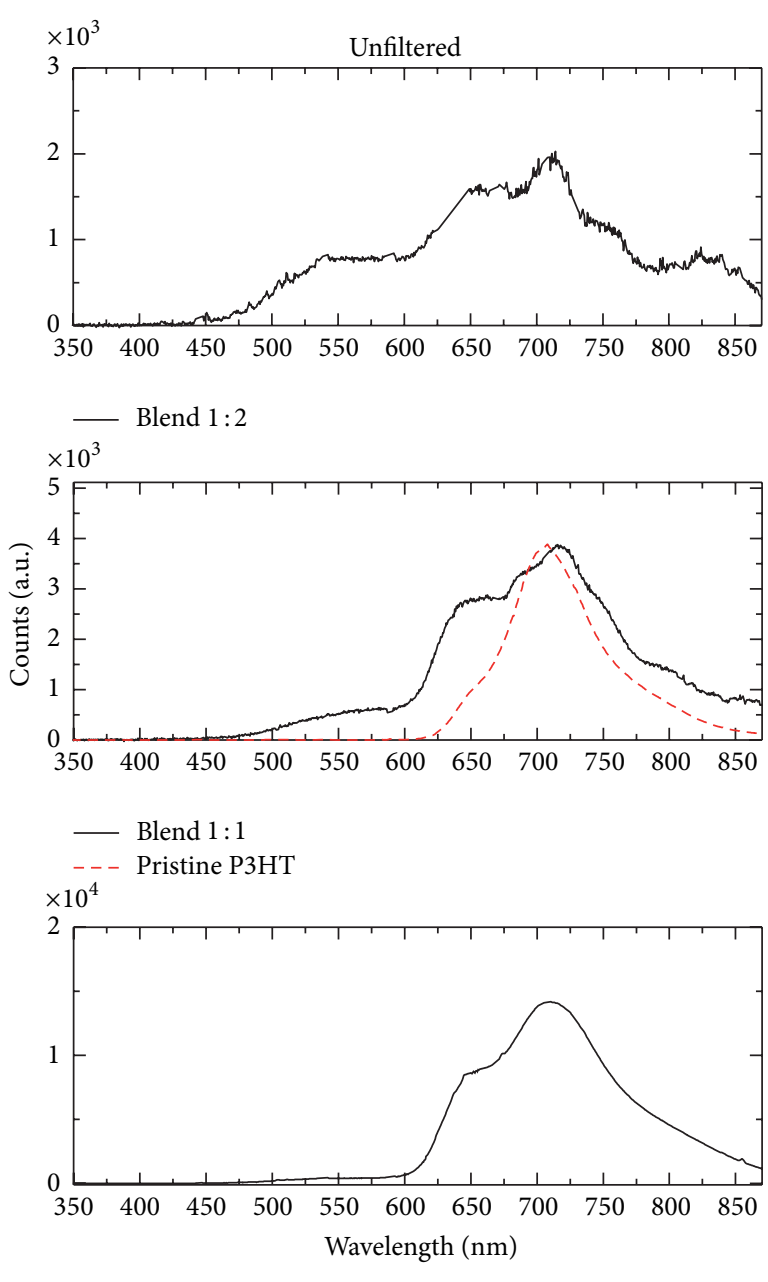

— Blend 2:1

FIGURE 1: Photoluminescence spectra of (from the top) $1: 2,1: 1$, and $2: 1 \mathrm{P} 3 \mathrm{HT}$ : PCBM unfiltered samples (increasing P3HT relative content). The data have been normalized to the sample thicknesses.

the contrary, the P3HT emission is clearly evident in the $2: 1$ sample.

Filtered Samples. PL spectra of filtered samples are reported in Figure 2.

The main feature that is clear from the spectral shape is still the presence of the band around $550 \mathrm{~nm}$ with non negligible intensity relative to the P3HT emission, similar to the one of the unfiltered samples, previously assigned to $\mathrm{CT}$ emission from PCBM clusters. These results then suggest that some of the PCBM clusters responsible for the emission have dimensions lower than $0.45 \mu \mathrm{m}$ (the filter pore size). On the other side, the quenching effect appears to be less pronounced (compare the $1: 2$ and $1: 1$ spectra), that could be an indication that the filtering process mainly removed not well dissolved PCBM from the solution. However, the thickness of the 1:1 sample in this case is higher, thus, other possible morphological and photophysical effects on the overall PL intensity can not be excluded. However, the main scope of PL measurements here is to give strong support 

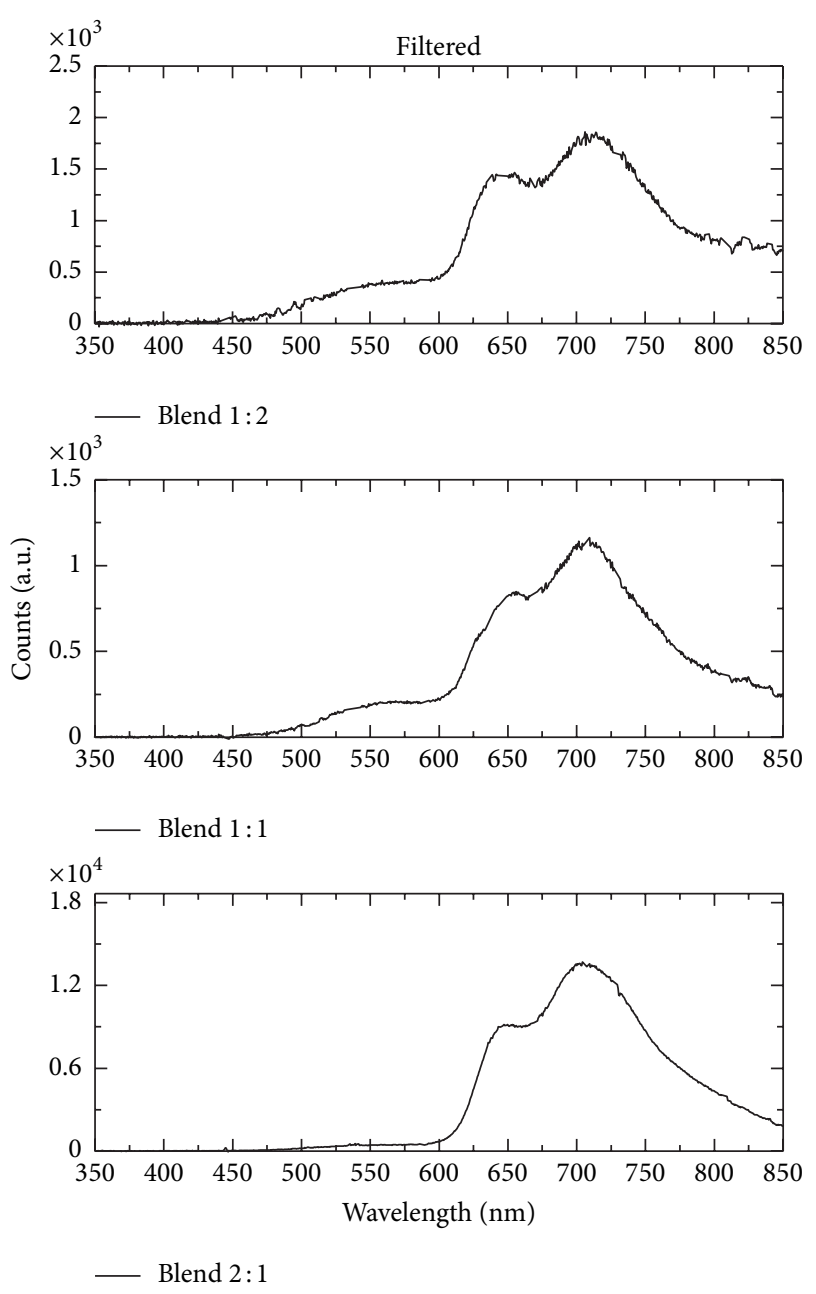

FIgUre 2: Photoluminescence spectra of (from the top) $1: 2,1: 1$, and 2:1 P3HT: PCBM filtered samples (increasing P3HT relative content). The data have been normalized to the sample thicknesses.

to the following conclusions about the phase origin of the detected signals during the CLSM experiments.

3.2. Confocal Laser Scanning Microscopy. In order to check for possible differences between the sample surfaces (air/blend or glass/blend interfaces) it is necessary that the instrument can section the observed specimen, therefore avoiding optical overlap of signals detected on the two surface planes. According to the Nikon instrument specification and to the general confocal laser microscopy principles [24, 25], the actual calculated total depth of field of the used system is $0.64 \mu \mathrm{m}$ (microscope pinhole was set to 1.2 Airy Unit). This means that there is no optical overlap between the regions at the two interfaces during the measurements (the optical slices on both sides do not overlap each other when the sample interfaces are on focus).

The collecting filters have been chosen in order to separate the emission from the two material phases. The $530 \mathrm{~nm}$ band pass filter allows to collect the emission generating from the PCBM rich phase only since there is no emission of P3HT below $\sim 600 \mathrm{~nm}$ (see Figures 1 and 2), while the $770 \mathrm{~nm}$ high pass filter should account mainly for the P3HT rich phase emission (the very weak PCBM emission around $700-750 \mathrm{~nm}$ [21] is cut off by the filter). The PL counts have been integrated over the whole images for the total signal intensity comparison in the following discussion. The reported confocal images in the following have been processed with a median filter for signal noise removing. No normalization has been performed.

Figure 3 reports the confocal images (collected at $770 \mathrm{~nm}$ and above) of the unfiltered samples (the important parameter in this case is the depth of field of $0.640 \mu \mathrm{m}$. This means that the microscope can collect without any overlap the light that comes from the focus plane \pm a slice of $320 \mathrm{~nm}$ thick).

Looking at the confocal images at $770 \mathrm{~nm}$ channel (Figure 3), where only the P3HT signal is expected, it is observed that for the $1: 2$ and $1: 1$ sample, the total signal intensity (PL counts integrated over the image area) at the glass/blend interface is lower than that at the air/blend interface, confirming a P3HT-phase preferential distribution on top of the specimen. The $1: 2$ and the $1: 1$ samples show at $530 \mathrm{~nm}$ (Figure 4) distinguishable PCBM signal at the glass/blend interface and a very weak signal at the air/blend interface, confirming a preferential arrangement of the PCBM phase on the bottom of the sample.

The averaged signal intensity @770 nm is on the contrary less different between the two interfaces for the (P3HTricher) 2:1 sample, meaning that the P3HT span all over both surfaces. On the contrary, looking to the total PL signal counts at the $530 \mathrm{~nm}$ channel (Figure $4(\mathrm{~g})$ ), where only PCBM-originating emission is expected, the signal is very low on both surfaces. This indicates that most of the PCBM emission could be absorbed by the overwhelming P3HT phase (see also the very weak PL @530 in Figure 1).

We can exclude a possible degradation of the polymer blend at the air/blend interface (respect to the glass/blend interface) due to oxygen interaction with the materials with potential photooxidation process - influencing the luminescence signal intensity. In fact, the oxygen diffusion constant in the blend is in the range $0.15-1.2 \mu \mathrm{m}^{2} \mathrm{~s}^{-1}$ [26-28], that means that oxygen penetrate the whole films studied here in 1-2 seconds up to the glass/blend interface. Thus, possible influences on luminescence should be the same on both surfaces. Moreover, the P3HT emission is not decreased by oxygen exposure in the $700 \mathrm{~nm}$ and above spectral region [26]. Furthermore, the ultrathin cover glass slice transmits more than $90 \%$ of the visible light (transmission spectrum measured by a Cary Varian 500 spectrophotometer, not shown). We can, therefore, neglect any possible glass effects on the blend absorption of laser pump on both interfaces as well as on the transmitted luminescence. Finally, we ruled out any optical interference since the glass thickness is by far greater than the pump and emission wavelength.

We point out that the phase preferential arrangement at the interfaces is a process driven by the differences in the surface energy between P3HT and PCBM (being the P3HT one smaller than that of PCBM [8]) and by the interaction of the blend with the substrate and the air $[29,30]$. Therefore, 


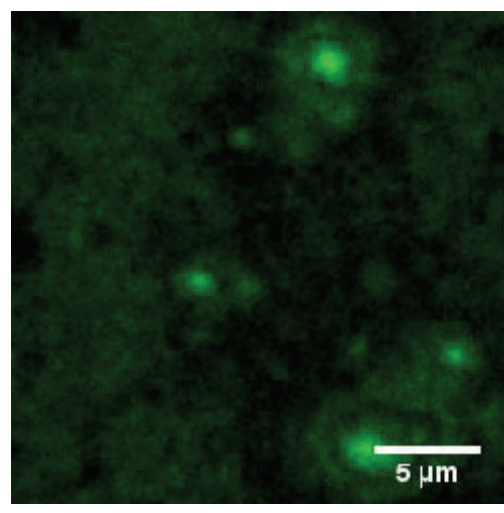

(a)

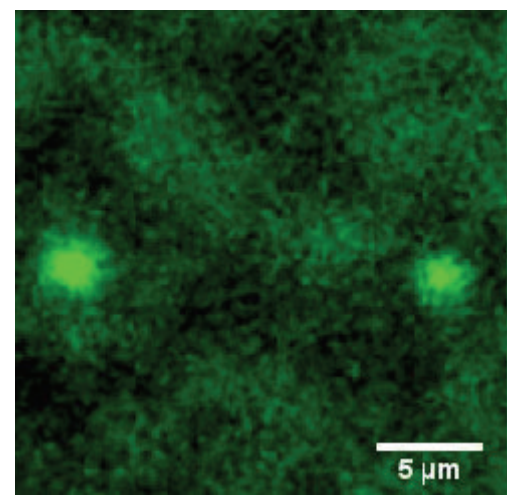

(c)

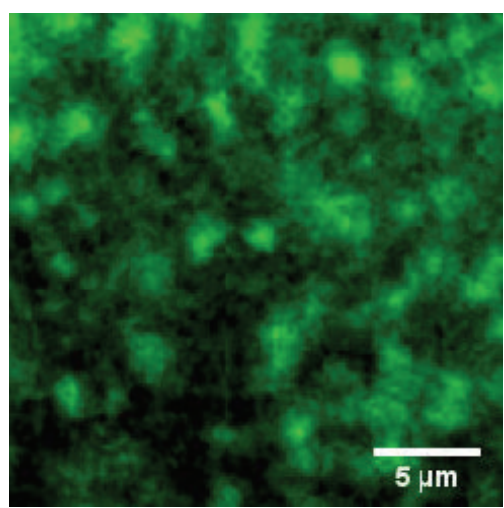

(e)

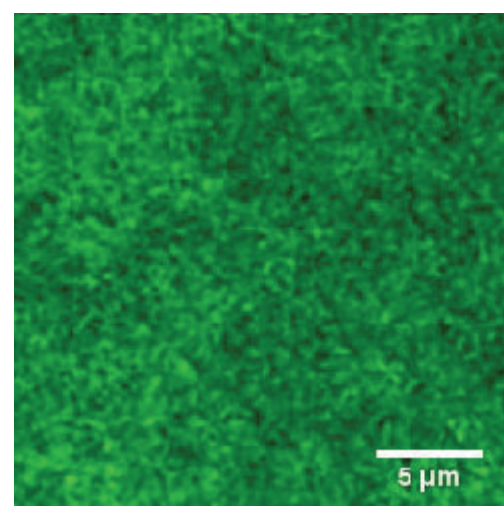

(b)

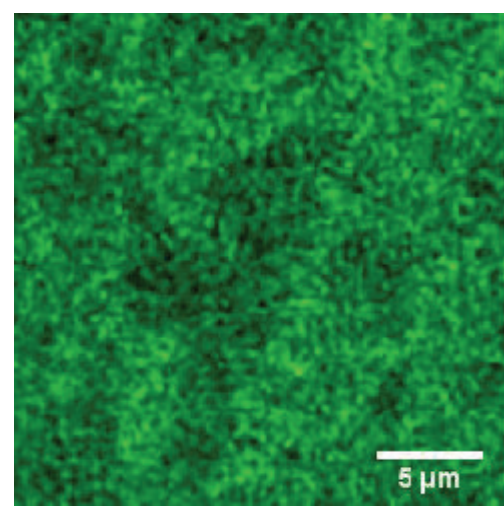

(d)

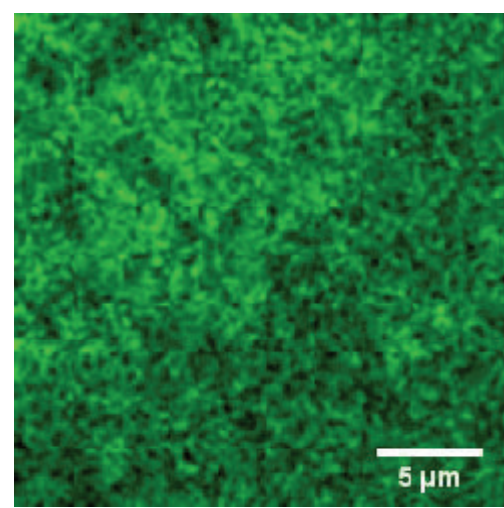

(f)

FiguRE 3: Confocal images of as casted samples: (a) and (b) 1:1 glass/blend interface and blend/air interface, respectively; (c) and (d) 1:2 glass/blend interface and blend/air interface, respectively; (e) and (f) $2: 1$ glass/blend interface and blend/air interface, respectively, images are $23.04 \times 23.04 \mu \mathrm{m}$ collected @770 nm (resolution: $0.09 \mu \mathrm{m} / \mathrm{px}, 512 \times 512 \mathrm{px}$ ) (color online).

the possible thickness variation among the samples, in the present range limit greater than $1 \mu \mathrm{m}$, does not influence the phase composition at the two interfaces [31]. Moreover, the scope of this work is to provide a fast detection of different phase behavior at the interfaces, not claimingon the contrary-to provide a tool for a complete vertical gradient segregation, since the layer thickness is too low for a convenient multiple slicing of the system. In our opinion, this is not a limitation if we consider the aim of this work, that is, surface phases characterization. It is possible that multiple stratifications could occur but, taking into account the observations given in the introduction about the dept of field and the optical slice overlap, it appears evident that, even in the case of a multilayered structure, the main contribution to the signal would come from the prevalent phase in the interface subsystem.

In order to investigate the dependence of the phase separation on possible PCBM aggregates/clusters content, we have repeated the experiments on fresh films deposited after filtering the solutions. 


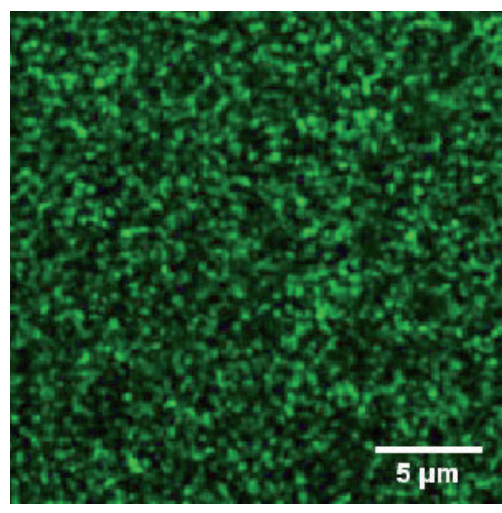

(a)

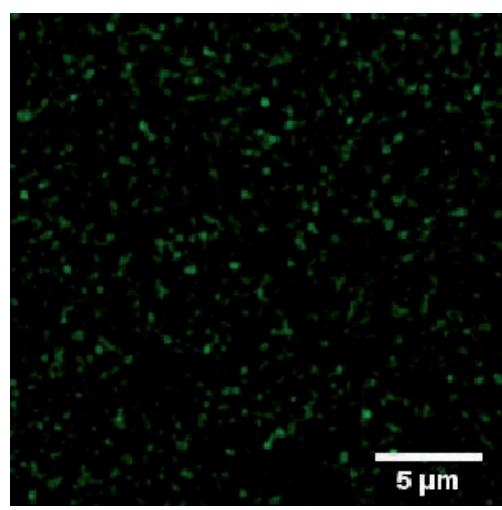

(d)

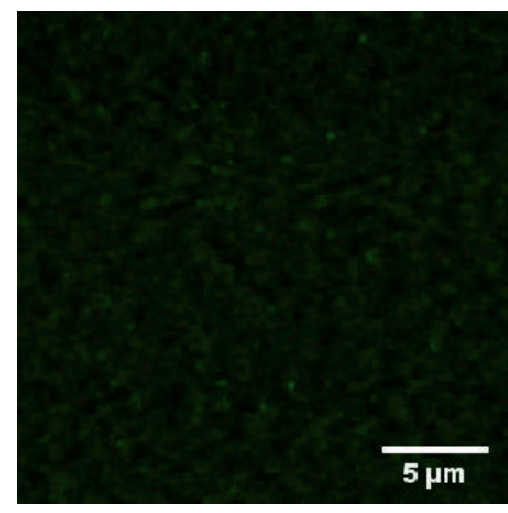

(b)

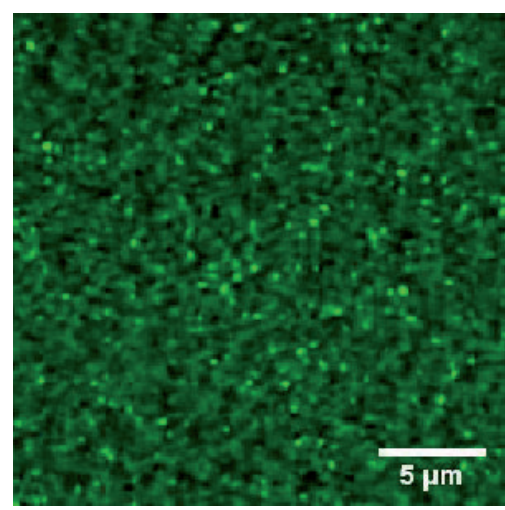

(e)

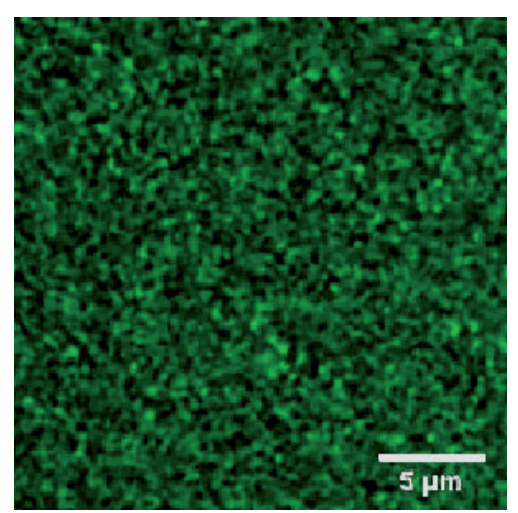

(c)

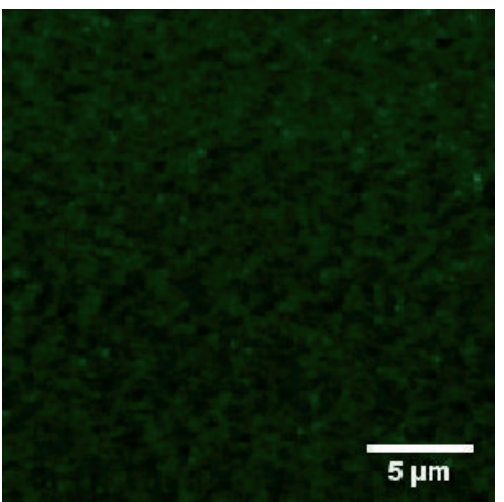

(f)

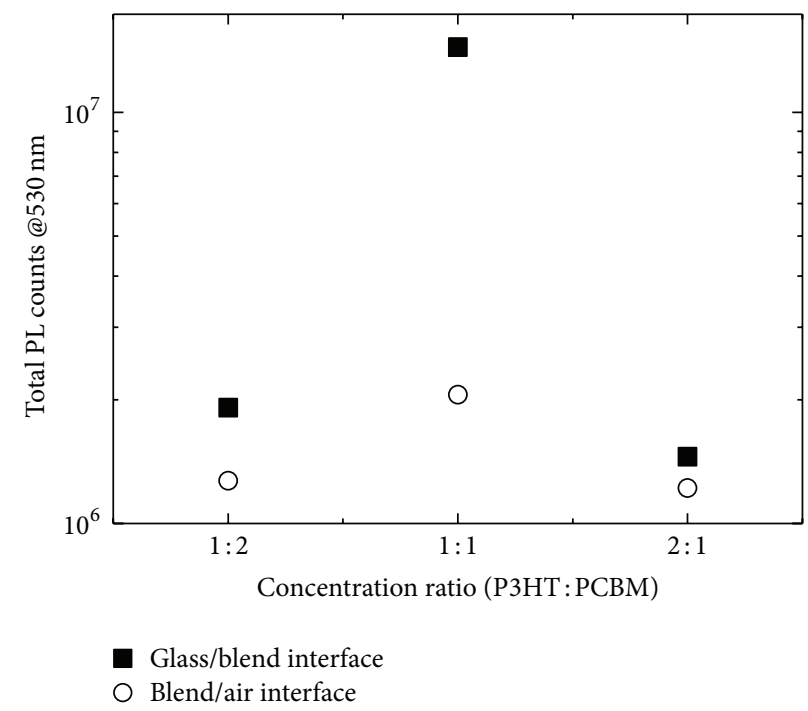

(g)

FIGURE 4: Confocal images collected @530 nm of as casted unfiltered samples: (a) and (b) 1:1 glass/blend interface and blend/air interface, respectively; (c) and (d) 1:2 glass/blend interface and blend/air interface, respectively; (e) and (f) $2: 1$ glass/blend interface and blend/air interface, respectively $(23.04 \times 23.04 \mu \mathrm{m}, 0.09 \mu \mathrm{m} / \mathrm{px}, 512 \times 512 \mathrm{px})$ (color online). (g) The corresponding total PL counts (total counts integrated all over the image area) as detected by the instrument.

Figure 5 reports the confocal images collected at $770 \mathrm{~nm}$ (glass/blend interfaces (a), (c), and (e), air/blend interface (b), (d), and (f)).

It is clear that there is a macroscopic micrometer-sized phase separation for all the samples at the glass/blend interface, where the brighter regions (percentage of signal higher than the average is of $33.20 \%, 41.82 \%$, and $34.62 \%$, respectively, calculated by digital image analysis) should be richer in polymer. On the contrary, no clear macroscopic structures are visible on the air/blend interface, that shows 


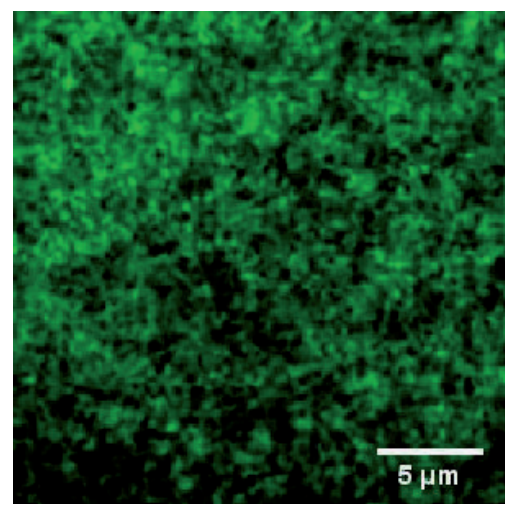

(a)

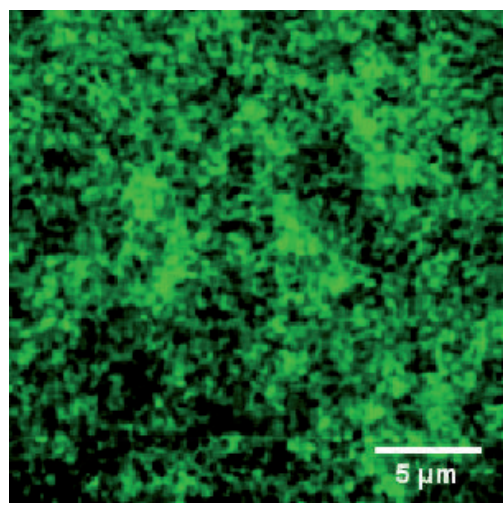

(c)

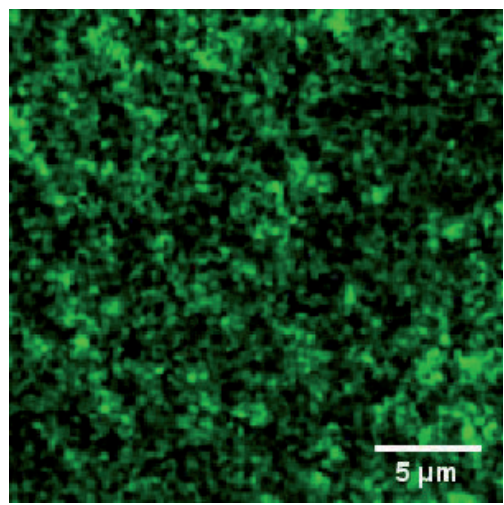

(e)

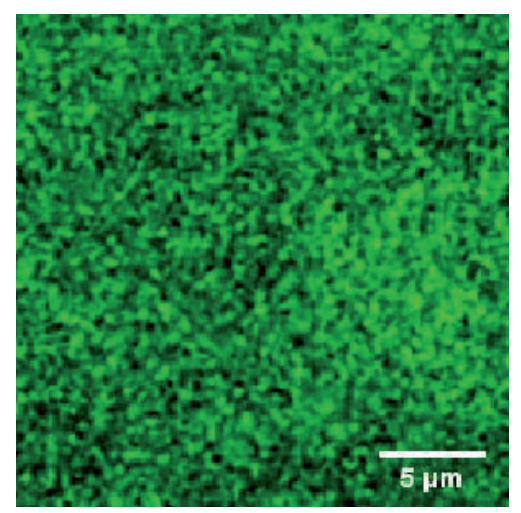

(b)

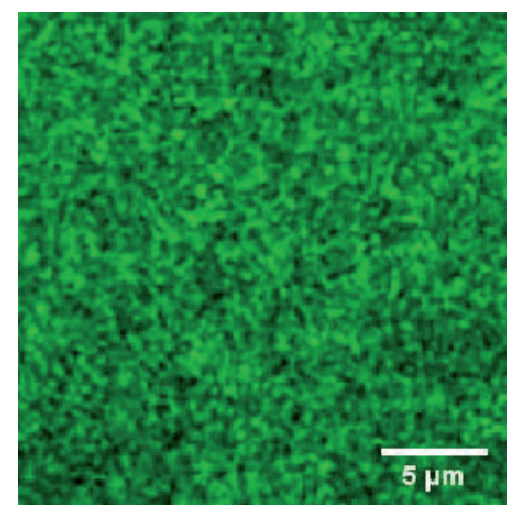

(d)

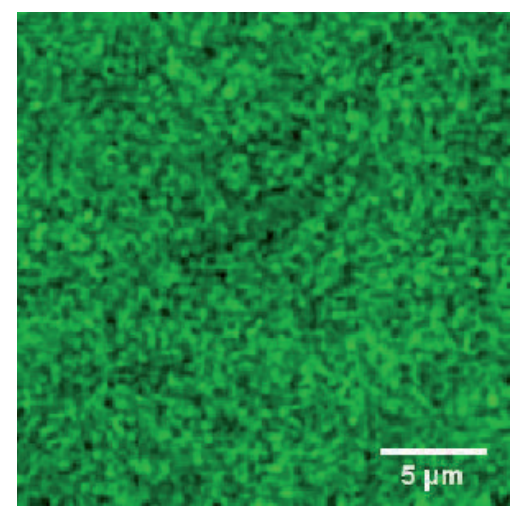

(f)

FIGURE 5: Confocal images of samples casted by filtering the initial solutions: (a) and (b) 1:1 glass/blend interface and blend/air interface, respectively; (c) and (d) 1:2 glass/blend interface and blend/air interface, respectively; (e) and (f) $2: 1$ glass/blend interface and blend/air interface, respectively; images are $23.04 \times 23.04 \mu \mathrm{m}$ collected @770 nm (resolution: $0.09 \mu \mathrm{m} / \mathrm{px}, 512 \times 512 \mathrm{px}$ ) (color online).

a quite uniform luminescence coming from $\mathrm{P} 3 \mathrm{HT}$ all over the surface, with almost no difference among the samples (for all, a coverage higher than $85 \%$ over the average signal intensity is calculated). Again, what is to be noted here is the averaged PL counts differences between the two interfaces. The PL intensity at the air/blend interfaces is indeed higher than that at the glass/blend interfaces, implying that there is a majority of $\mathrm{P} 3 \mathrm{HT}$ on the top surface.

The confocal images in the PCBM aggregates emission range @530 nm (Figure 6) show again more PCBM PL signal at the glass/blend interface, while the signal at the air/blend interface is definitively lower (see PL counts plot in the same figure). The fullerene phase lies mainly at the bottom of the sample.

From the results on filtered samples, we can conclude that the phase composition at the interfaces does not depend on possible clusters/agglomerates of one of the materials. These results demonstrate that the differences in the phase composition at the two interfaces can be revealed by CLSM by comparing the PL intensity even in fine-mixed samples that do not show any particular big cluster or evident macroscopic features. 


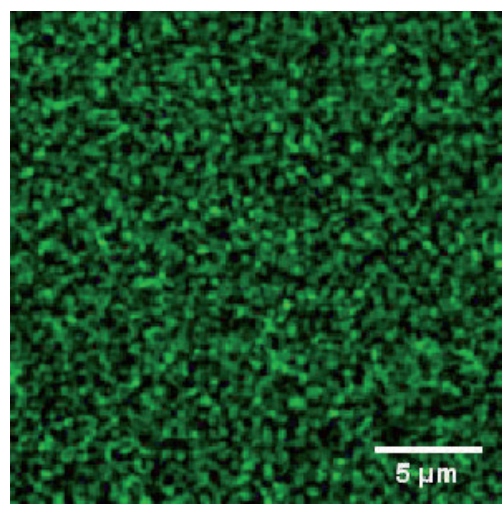

(a)

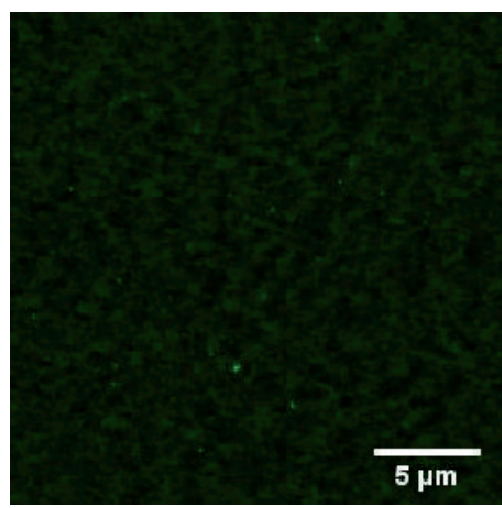

(d)

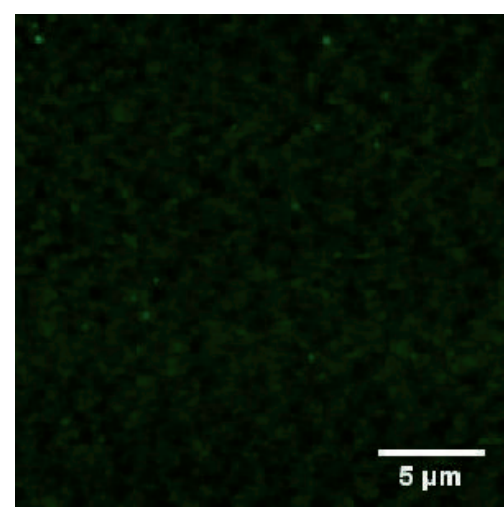

(b)

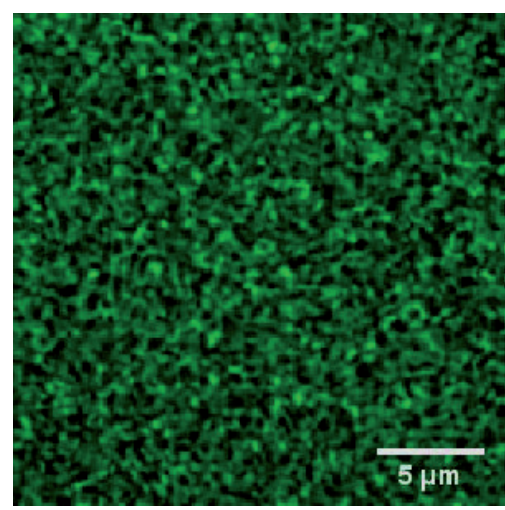

(e)

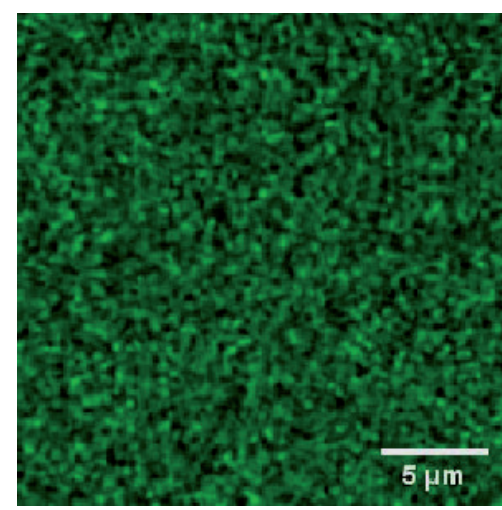

(c)

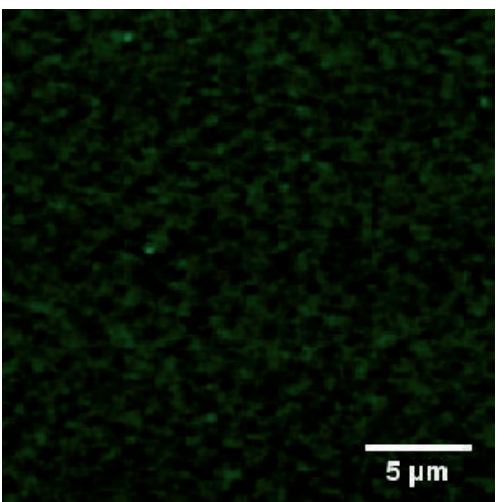

(f)

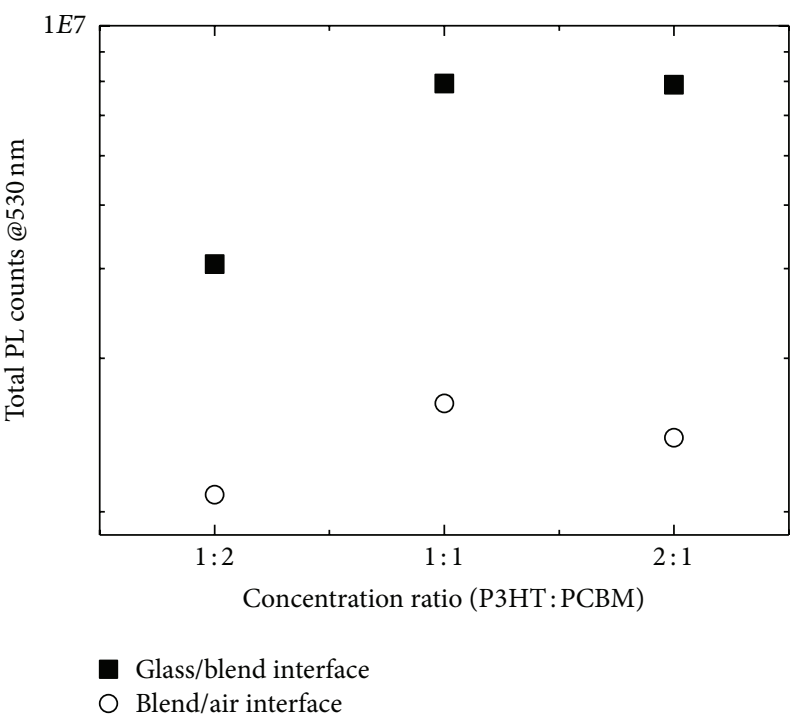

(g)

FIGURE 6: Confocal images collected @530 nm of filtered samples: (a) and (b) 1:1 glass/blend interface and blend/air interface, respectively; (c) and (d) 1:2 glass/blend interface and blend/air interface, respectively; (e) and (f) $2: 1$ glass/blend interface and blend/air interface, respectively $(23.04 \times 23.04 \mu \mathrm{m}, 0.09 \mu \mathrm{m} / \mathrm{px}, 512 \times 512 \mathrm{px})$ (color online). (g) The corresponding total PL counts (total counts integrated all over the image area) as detected by the instrument.

Although the presented results are related to a P3HT : PCBM thick film casted on glass, the used technique is generally valid for different thick films. The conclusions are valid even when thinking to a structure geometry in which the upper layer of the substrate is composed, for example, by commonly used materials like PEDOT:PSS or $\mathrm{ZnO}$ or $\mathrm{TiO}_{2}$ when realizing diode-like structures. Indeed, even if it is not possible to directly perform the here reported CLSM 
experiment comprising such additional layers-because the photoluminescence counts detected at the two interfaces would no more be comparable, since the surface comprising the additional layers would suffer from increased scattering and reduced transmittance-in the limits of the present experiment, the parameter that has a strong influence on the interface morphology is the hydrophilic or hydrophobic nature of the substrates interacting with the blend [29]. It is known that the PCBM tends to accumulate towards hydrophilic substrates [30] as confirmed by our results as the standard glass is hydrophilic. The accumulation would then be even more pronounced if instead of on glass the blend was casted on the high hydrophilic PEDOT: PSS or on the hydrophilic $\mathrm{ZnO}$ or $\mathrm{TiO}_{2}\left(\mathrm{ZnO}\right.$ and $\mathrm{TiO}_{2}$ can be easily made hydrophilic by simple UV irradiation $[32,33])$. Since many blend systems used in the cited application fields are composed by species with different hydrophobic/philic properties and free surface energies, the generality of our reports is evident. All the results cited in the Introduction about the vertical phase stratification have been obtained using experimental techniques and facilities not commonly available and that require a great expertise and timeconsuming implementation. Moreover, at this stage (to just know if there is a majority of one phase on one particular interface), it is not mandatory to have a $\mathrm{nm}$ scale resolution along the sample plane: the important parameter to be detected is the average content on one surface of one of the materials and not the nm scale fine structure of the blend.

\section{Conclusions}

The Confocal Laser Scanning Microscopy has been exploited as a common, simple, fast, and user-friendly technique to characterize the phase composition differences at interfaces of polymer blends. A clear difference in the macroscopic phase content at the bottom and at the top of the samples has been detected. The novel idea of this work is to apply the CLSM to thick films in fields where the effects of external interfaces are important.

Even if the results on thick films can not be extended directly on thinner films and consequently to those application that require thin films, these results are certainly useful in the preliminary blend morphology characterization field when the phase content at the interfaces is an important parameter to be considered and the thickness of the layer must be of few micrometers. Using the CLSM instead of other techniques like XRD, XPS, UPS, SIMS, or Neutron Scattering could indeed allow a fast in-lab checking of the influence of mixing ratio, solvent, annealing, and preparation process in a quick few-step experiment. Experiments on different and more interesting systems as well as experiments to compare more precisely the morphology of both phases of various blend systems are actually in progress.

\section{Conflict of Interests}

The authors declare that there is no conflict of interests regarding the publication of this paper.

\section{Acknowledgments}

Dr. Mauro Lomascolo and Dr. Roberto Rella of IMMCNR of Lecce are kindly acknowledged for providing the photoluminescence experimental setup, the chemical fume hood, and the spectrophotometer. Professor Massimo Di Giulio is kindly acknowledged for providing the profilometer. Sandro Lattante has received financial support from the FP7 ALHsolar project-SP3-People Marie Curie ERG (PERG04GA-2008-239342). This work has been supported by Regione Puglia through the projects Sens\&MicroLAB and SMILE.

\section{References}

[1] C. M. Björström, S. Nilsson, A. Bernasik et al., "Vertical phase separation in spin-coated films of a low bandgap polyfluorene/PCBM blend-Effects of specific substrate interaction," Applied Surface Science, vol. 253, no. 8, pp. 3906-3912, 2007.

[2] C. M. Björström, A. Bernasik, J. Rysz et al., "Multilayer formation in spin-coated thin films of low-bandgap polyfluorene:PCBM blends," Journal of Physics Condensed Matter, vol. 17, no. 50, pp. L529-L534, 2005.

[3] H. Lu, B. Akgun, and T. P. Russel, "Morphological characterization of a low-bandgap crystalline polymer: PCBM bulk heterojunction solar cells," Advanced Energy Materials, vol. 1, pp. 870-878, 2011.

[4] P. A. Staniec, A. J. Parnell, and A. D. F. Dunbar, "The nanoscale morphology of a PCDTBT:PCBM photovoltaic blend," Advanced Energy Materials, vol. 1, pp. 499-504, 2011.

[5] K.-H. Yim, Z. Zheng, R. H. Friend, W. T. S. Huck, and J.-S. Kim, "Surface-directed phase separation of conjugated polymer blends for efficient light-emitting diodes," Advanced Functional Materials, vol. 18, no. 19, pp. 2897-2904, 2008.

[6] W. C. Tsoi, S. J. Spencer, L. Yang et al., "Effect of crystallization on the electronic energy levels and thin film morphology of P3HT:PCBM blends," Macromolecules, vol. 44, no. 8, pp. 29442952, 2011

[7] B. Xue, B. Vaughan, C.-H. Poh et al., "Vertical stratification and interfacial structure in P3HT:PCBM Organic solar cells," Journal of Physical Chemistry C, vol. 114, no. 37, pp. 15797-15805, 2010.

[8] P. G. Karagiannidis, D. Georgiou, C. Pitsalidis, A. Laskarakis, and S. Logothetidis, "Evolution of vertical phase separation in P3HT:PCBM thin films induced by thermal annealing," Materials Chemistry and Physics, vol. 129, no. 3, pp. 1207-1213, 2011.

[9] M. V. Madsen, K. O. Sylvester-Hvid, B. Dastmalchi et al., "Ellipsometry as a nondestructive depth profiling tool for rollto-roll manufactured flexible solar cells," Journal of Physical Chemistry C, vol. 115, no. 21, pp. 10817-10822, 2011.

[10] A. J. Moule, J. B. Bonekamp, and K. Meerholz, "The effect of active layer thickness and composition on the performance of bulk-heterojunction solar cells," Journal of Applied Physics, vol. 100, Article ID 094503, 2006.

[11] T. N. Ng, W. S. Wong, R. A. Lujan et al., "Characterization of flexible image sensor arrays with bulk heterojunction organic photodiodes," in Organic Field-Effect Transistors VII and Organic Semiconductors in Sensors and Bioelectronics, Z. Bao, I. McCulloch, R. Shinar, and G. G. Malliaras, Eds., vol. 7054 of Proceedings of SPIE, August 2008. 
[12] L. L. Lavery, G. L. Whiting, and A. C. Arias, "All ink-jet printed polyfluorene photosensor for high illuminance detection," Organic Electronics: Physics, Materials, Applications, vol. 12, no. 4, pp. 682-685, 2011.

[13] J. Mysliwiec, L. Sznitko, S. Bartkiewicz et al., "Amplified spontaneous emission in the spiropyran-biopolymer based system," Applied Physics Letters, vol. 94, no. 24, Article ID 241106, 2009.

[14] J. Mysliwiec, L. Sznitko, A. Sobolewska, S. Bartkiewicz, and A. Miniewicz, "Lasing effect in a hybrid dye-doped biopolymer and photochromic polymer system," Applied Physics Letters, vol. 96, no. 14, Article ID 141106, 2010.

[15] M. Anni, A. Perulli, and G. Monti, “Thickness dependence of the amplified spontaneous emission threshold and operational stability in poly(9,9-dioctylfluorene) active waveguides," Journal of Applied Physics, vol. 111, Article ID 093109, 2012.

[16] V. Navarro-Fuster, I. Vragovic, E. M. Calzado et al., "Film thickness and grating depth variation in organic second-order distributed feedback lasers," Journal of Applied Physics, vol. 112, Article ID 043104, 2012.

[17] E. M. Calzado, M. G. Ramirez, P. G. Boj, and M. A. Diaz-Garcia, "Thickness dependence of amplified spontaneous emission in low-absorbing organic waveguides," Applied Optics, vol. 51, pp. 3287-3293, 2012.

[18] J. Y. Oh, W. S. Jang, T. I. Lee, J.-M. Myoung, and H. K. Baik, "Driving vertical phase separation in a bulk-heterojunction by inserting a poly(3-hexylthiophene) layer for highly efficient organic solar cells," Applied Physics Letters, vol. 98, Article ID 023303, 2011.

[19] A. D. F. Dunbar, P. Mokarian-Tabari, A. J. Parnell, S. J. Martin, M. W. A. Skoda, and R. A. L. Jones, "A solution concentration dependent transition from self-stratification to lateral phase separation in spin-cast PS:d-PMMA thin films," European Physical Journal E, vol. 31, no. 4, pp. 369-375, 2010.

[20] B. Gholamkhass, T. J. Peckham, and S. Holdcroft, "Poly(3hexylthiophene) bearing pendant fullerenes: aggregation vs. self-organization," Polymer Chemistry, vol. 1, no. 5, pp. 708-719, 2010.

[21] S. Cook, H. Ohkita, Y. Kim, J. J. Benson-Smith, D. D. C. Bradley, and J. R. Durrant, "A photophysical study of PCBM thin films," Chemical Physics Letters, vol. 445, no. 4-6, pp. 276-280, 2007.

[22] C. J. Brabec, G. Zerza, G. Cerullo et al., “Tracing photoinduced electron transfer process in conjugated polymer/fullerene bulk heterojunctions in real time," Chemical Physics Letters, vol. 340, no. 3-4, pp. 232-236, 2001.

[23] F. Padinger, R. S. Rittberger, and N. S. Sariciftci, "Effects of postproduction treatment on plastic solar cells," Advanced Functional Materials, vol. 13, no. 1, pp. 85-88, 2003.

[24] S. Wilhelm, B. Grobler, M. Gluch, and H. Heinz, Confocal Laser Scanning Microscopy: Principles, 2003.

[25] R. H. Webb, "Confocal optical microscopy," Reports on Progress in Physics, vol. 59, p. 427, 1996.

[26] A. Sperlich, H. Kraus, C. Deibel, H. Blok, J. Schmidt, and V. Dyakonov, "Reversible and irreversible interactions of poly(3hexylthiophene) with oxygen studied by spin-sensitive methods," Journal of Physical Chemistry B, vol. 115, no. 46, pp. 1351313518, 2011.

[27] M. S. A. Abdou, F. P. Orfino, Y. Son, and S. Holdcroft, "Interaction of oxygen with conjugated polymers: charge transfer complex formation with poly(3-alkylthiophenes)," Journal of the American Chemical Society, vol. 119, no. 19, pp. 4518-4524, 1997.
[28] L. Lüer, H.-J. Egelhaaf, D. Oelkrug et al., "Oxygen-induced quenching of photoexcited states in polythiophene films," Organic Electronics: Physics, Materials, Applications, vol. 5, no. 1-3, pp. 83-89, 2004.

[29] G. Li Destri, T. F. Keller, M. Catellani, F. Punzo, K. D. Jandt, and G. Marletta, "Interfacial free energy Driven nanophase separation in poly(3-hexylthiophene)/[6,6]-phenyl-C61-butyric acid methyl ester thin films," Langmuir, vol. 28, no. 11, pp. 5257-5266, 2012.

[30] Z. Xu, L. Chen, and G. Yang, "Vertical phase separation in poly(3-hexylthiophene): fullerene derivative blends and its advantage for inverted structure solar cells," Advanced Functional Materials, vol. 19, pp. 1227-1234, 2009.

[31] H. Ogawa, T. Kanaya, K. Nishida, and G. Matsuba, "Phase separation and dewetting in polystyrene/poly(vinyl methyl ether) blend thin films in a wide thickness range," Polymer, vol. 49, no. 1, pp. 254-262, 2008.

[32] T. Charinpanitkul, W. Suthabanditpong, H. Watanabe et al., "Improved hydrophilicity of zinc oxide-incorporated layer-bylayer polyelectrolyte film fabricated by dip coating method," Journal of Industrial and Engineering Chemistry, vol. 18, no. 4, pp. 1441-1445, 2012.

[33] V. Kekkonen, A. Hakola, T. Kajava et al., "Self-erasing and rewritable wettability patterns on $\mathrm{ZnO}$ thin films," Applied Physics Letters, vol. 97, no. 4, Article ID 044102, 2010. 

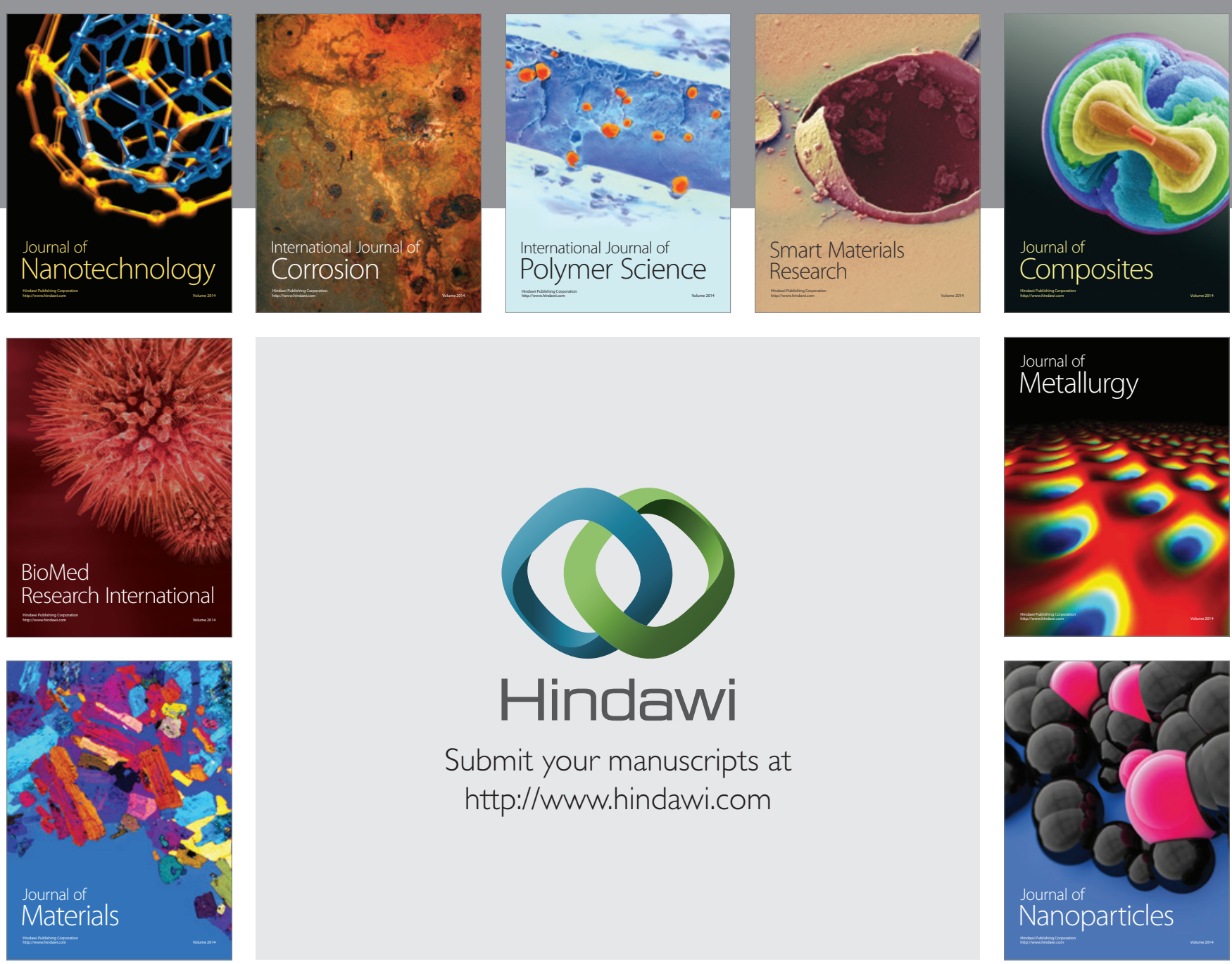

Submit your manuscripts at http://www.hindawi.com
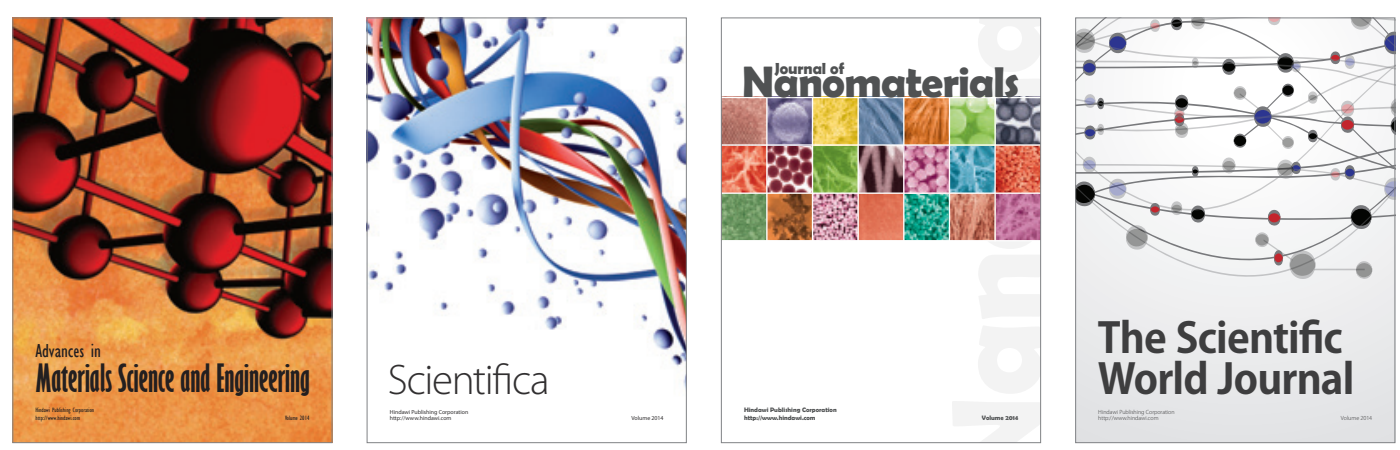

\section{The Scientific World Journal}
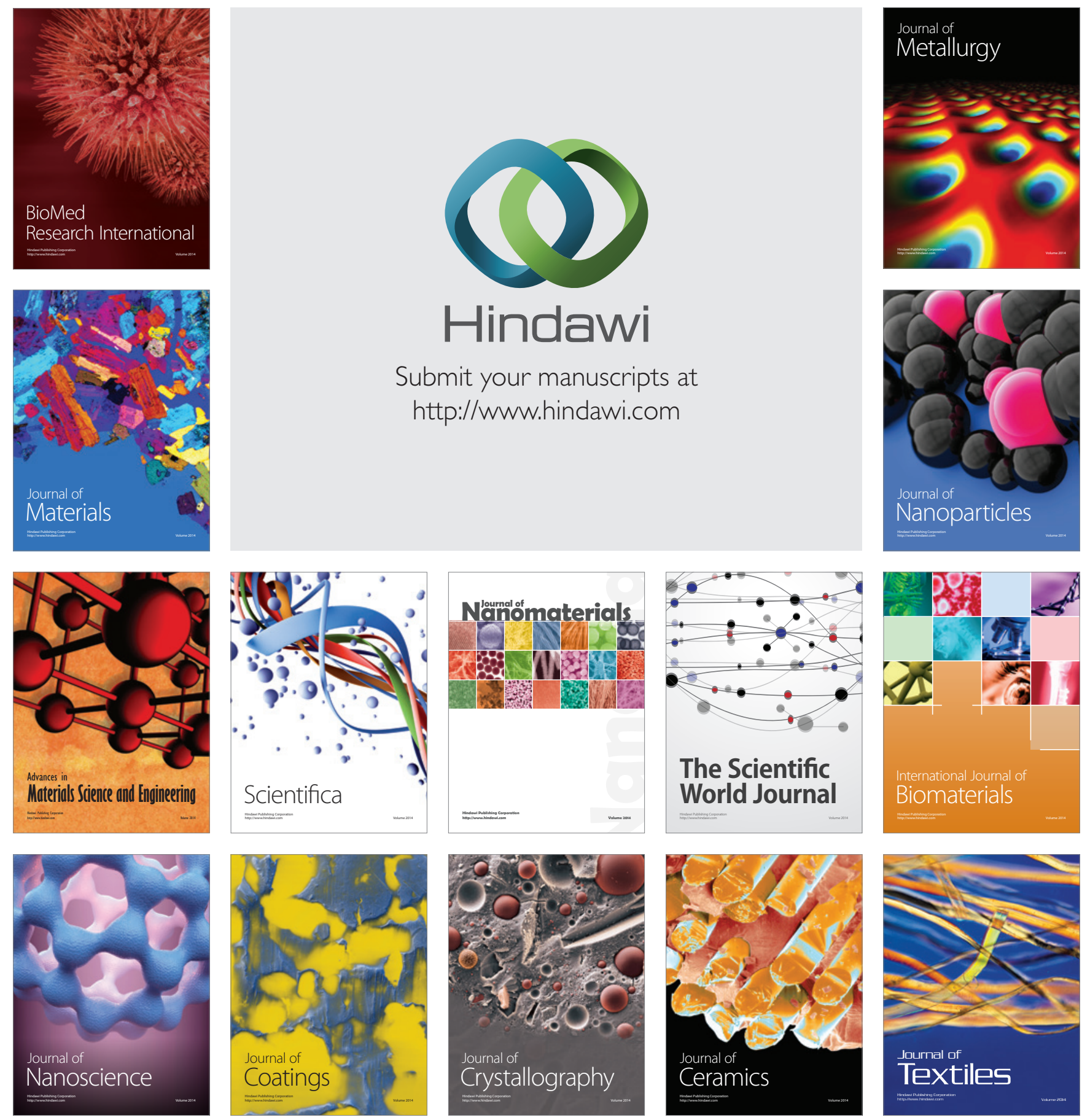